\title{
A new many-body wave function for BCS-BEC crossover in Fermi gases
}

\author{
Shina Tan and K. Levin \\ James Franck Institute and Department of Physics, University of Chicago, Chicago, Illinois 60637
}

\begin{abstract}
We present a new many body formalism for BCS-BEC crossover, which represents a modification of the BCS-Leggett ground state to include 4-fermion, and higher correlations. In the BEC regime, we show how our approach contains the Petrov et al 4-fermion behavior and associated scattering length $a_{d d}$ at short distances, and secondly reduces to composite-boson Bogoliubov physics at long distances. It reproduces the Lee-Yang term, whose numerical value is also fixed by $a_{d d}$. We have also examined the next term beyond the LeeYang correction in a phenomenological fashion, building on cloud size data and collective mode experiments. However, one has to view this phenomenological analysis with some caution since experiments are in a state of flux and are performed close to unitarity.

PACS numbers: 03.75.Ss
\end{abstract}

Keywords: BCS-BEC crossover; atomic fermi gas; ground state; collective excitation

There have been exciting new developments in the field of trapped fermionic atoms [1, 2, 3] in which applied magnetic fields are used to effect a transition from BCS superfluidity to Bose Einstein condensation (BEC). The simplest mean field wave function [4, 5] which describes these phenomena at $T=0$ is, effectively, the BCS wave function $\left|\psi_{\mathrm{BCS}}\right\rangle$ with self consistently determined (fermionic) chemical potential in the presence of pairing interactions of arbitrary strength. This has met with some experimental success [6] but it is clearly lacking in the sense that it does not incorporate the exact twomolecule (4-fermion) scattering physics [7]. In this mean field theory, the 4-fermion interaction, parametrized by the dimerdimer scattering length $a_{d d}$, is overestimated by a factor of 3 , leading to a similar overestimate in the cloud size [8]. Condensate depletion is also absent in this lowest order mean-field theory.

This earlier work raises a fundamental question which we address here. How does one arrive at a many body wave function which incorporates the few body constraints and what are its implications for experiment. While, there have been efforts [9, 10] to go beyond the leading order mean field theory in the literature, there has never been a demonstrated consistency between the exact few body constraints and N-body behavior. Our work is the first to make these two points of view compatible and in the process, introduces a new perturbation scheme which replaces the usual Feynmann diagrammatic expansion. Here we derive an equation of state for molecular bosons at the level of the Bogoliubov approximation, and, thereby set up a basis from which one can compute various properties of the gas. Of particular interest are those which are associated with the underlying fermionic physics, which are absent in a conventional BEC of true bosons.

The parameters appearing in our wave function are determined directly by an energy minimization procedure; we stress that there are no fitting parameters. The equation of state reduces to the well known Lee-Yang like formula [11], but with the scattering length $a_{d d}$ determined by the exact 4fermion physics [7]. Finally, our wave function appears compatible with the general features of experimental data on two different experiments: cloud sizes [8] and collective modes [12].

We begin by summarizing our results for the many body wave function and its consequences before providing the detailed methodology we employ. Additional details are given in the unpublished supporting material (Ref. [13]) which we have made available. One may rewrite the mean field wave function [4, 5] as

$$
\left|\psi_{\mathrm{BCS}}\right\rangle \propto \exp \left(\sum_{\mathbf{k}} \alpha_{\mathbf{k}} c_{\mathbf{k} \uparrow}^{\dagger} c_{-\mathbf{k} \downarrow}^{\dagger}\right)|0\rangle
$$

where $c_{\mathbf{k} \sigma}$ destroys fermions with momentum $\hbar \mathbf{k}$ and spin $\sigma$ [14], and $|0\rangle$ is the particle vacuum. Written in this way, the BCS wave function is seen to be the "composite-boson" version of the Gross-Pitaevskii (GP) wave function of conventional BEC.

It seems natural to presume that corrections to this GP-like wave function should capture some of the physics of the conventional Bogoliubov approach which provides an improvement over the GP wave function, appropriate to bosonic systems. We may rewrite the corresponding Bogoliubov wave function as

$$
\left|\psi_{\text {Bogoliubov }}\right\rangle=\exp \left(N_{0}^{1 / 2} b_{0}^{\dagger}+\sum_{\mathbf{q}>0} x_{\mathbf{q}} b_{\mathbf{q}}^{\dagger} b_{-\mathbf{q}}^{\dagger}\right)|0\rangle
$$

where $b_{\mathbf{q}}^{\dagger}$ is the creation operator of bosons with momentum $\hbar \mathbf{q}$ which, like $b_{0}^{\dagger}$, must be replaced by a bilinear form of fermion creation operators. In this way, one sees that four fermion terms are required to go beyond the simple BCS-like mean-field theory.

On this basis we return to Eq. (1) and write the natural correction terms. As will be shown below, to capture the physics of the deep BEC region, 6-fermion as well as 4-fermion corrections are needed. This goes beyond earlier work [9]. More generally one can contemplate a many body wave function of the form 


$$
|\psi\rangle=\exp \left(\frac{1}{2 !} \sum_{\mathbf{K}} \alpha_{\mathbf{K}} c_{\mathbf{K}}^{\dagger} c_{-\mathbf{K}}^{\dagger}+\frac{1}{4 !} \sum_{\mathbf{K}^{\prime} \mathbf{s}} \beta_{\mathbf{K}_{1} \mathbf{K}_{2} \mathbf{K}_{3} \mathbf{K}_{4}} c_{\mathbf{K}_{1}}^{\dagger} c_{\mathbf{K}_{2}}^{\dagger} c_{\mathbf{K}_{3}}^{\dagger} c_{\mathbf{K}_{4}}^{\dagger}+\frac{1}{6 !} \sum_{\mathbf{K}^{\prime} \mathbf{s}} \gamma_{\mathbf{K}_{1} \cdots \mathbf{K}_{6}} c_{\mathbf{K}_{1}}^{\dagger} \ldots c_{\mathbf{K}_{6}}^{\dagger}+\cdots\right)|0\rangle
$$

where each $\mathbf{K}_{i}$ represents a shorthand notation for $\mathbf{k}_{i} \sigma_{i}$, and $-\mathbf{K}$ refers to the fact that both the momentum and the spin are reversed. There are constraints imposed on the coefficients $\alpha$, $\beta$, $\gamma$, etc, which can be readily formulated [13], although we will not do so here.

We show below how to compute physical properties in terms of these wave function parameters. We do this in a fashion which is also consistent with Ref. [7]. Thus, to determine, for example, $a_{d d}$, the wave function is adjusted to minimize the energy, at a given fixed number of fermions. For general values of $k_{F} a$ this procedure is very difficult to implement. However, in the low-density regime, given more precisely by $n_{d} a_{d d}^{3} \ll 1$, higher-order correlations in the wave function are much weaker, and we are able to construct a systematic perturbation theory. For this reason, our theory is mostly limited to the deep BEC regime of the crossover $\left(n_{d} a_{d d}^{3} \ll 1\right)$. This is different from some other approaches which produce various smooth curves of the ground state energy in the whole BCS-BEC crossover [6, 15, 16]. Despite this limitation, physical observables (such as the equation of state) take the form of well-controlled low-density expansions, and the functional forms, as well as the coefficients of such expansions can be exactly determined within our theory, whereas the alternative theories [6, 15, 16] lack such exactness in the BEC limit.

The ground state expectation value of any physical observable $O$ is

$$
\langle O\rangle=\frac{\left\langle 0\left|\mathcal{T} O \exp \left(\alpha^{\dagger}+\beta^{\dagger}+\cdots+\alpha+\beta+\cdots\right)\right| 0\right\rangle}{\left\langle 0\left|\mathcal{T} \exp \left(\alpha^{\dagger}+\beta^{\dagger}+\cdots+\alpha+\beta+\cdots\right)\right| 0\right\rangle},
$$

where $\alpha, \beta$, etc, are the terms in the exponent of the right side of Eq. (3), and $\mathcal{T}$ is a "time-ordering" operator defined by assigning the fermion creation operators in $\alpha, \beta$, etc the earliest "time", the annihilation operators in $\alpha^{\dagger}, \beta^{\dagger}$, etc the latest "time". Finally, those times appearing in $O$ enter as intermediate "times" increasing monotonically from right to left. A standard Feynman diagrammatic method is then used to evaluate Eq. (4). It should be noted that, in our present approach, the terms in the wave function (instead of the interaction) serve as vertices, in contrast to the textbook applications of Feynman diagrams. Our approach is suited to the bosonic regime, where the attractive interaction between fermions is so strong that they form bound states.

One can contemplate very general interactions between two fermions which lead to these bound states. Under these circumstances, it follows from Eq. (3) that the ground state en- ergy is given by

$$
\begin{aligned}
E / \Omega=n_{d} E_{d}^{(0)}+2 \pi \hbar^{2} n_{d}^{2} a_{d d} / m_{d} & \\
\times & \left\{1+[128 /(15 \sqrt{\pi})] \sqrt{n_{d} a_{d d}^{3}}+\cdots\right\},
\end{aligned}
$$

which is similar to that of point-like bosons [11]. Here $\Omega$ is the volume, $n_{d}=n / 2$ is the number density of dimers, $m_{d}=2 m$ is the mass of the dimer, $m$ is the mass of each fermion, $E_{d}^{(0)}$ the energy of a zero-momentum dimer in vacuum, and $a_{d d}$ is the self consistently determined dimer-dimer scattering length. It should be stressed that this equation is very general; it applies even when the fermionic interaction is not a contact interaction. When the interaction has a finite range (comparable to the fermionic scattering length $a$ ), the famous calculation of Petrov et al in Ref. [7] needs to be corrected and $a_{d d} / a \neq 0.6$. It is the actual $a_{d d}$, determined from the solution to the quantum 4-fermion problem for the lowenergy scattering of two molecules [see Eq. 9 below], that must be used in Eq. (5). When the interaction is of very short range ( $\ll a)$ then the $a_{d d}$ in our theory reduces to that in Ref. [7].

An equation of state associated with the first two terms in Eq. (5) (between the curly brackets) was first proposed in Ref. [17], based on the assumption that fermionic dimers were governed by the same equation of state as point-like bosons. It is of considerable interest that we have confirmed this equation of state in the context of a fermionic system. Also note that this equation of state [Eq. [5] contains $n_{d} a_{d d}^{3} \ll 1$ as a small parameter [18]. While one might be inclined to consider a power series in $k_{F} a$, in this low density regime, where $k_{F} \propto n^{1 / 3}$ is the formal Fermi wave vector, we have shown that this is incorrect. When all fermions form tightly bound pairs, there is no Fermi sphere, and no natural expansion in terms of $n^{1 / 3}$ exists.

We now provide some details for our calculation by sketching how this wave function is determined in the BEC regime. If $n_{d} a_{d d}^{3} \ll 1$, higher correlation terms in the exponent in Eq. (3) are progressively less important. Their orders of magnitude are derived, determining which coefficients we should retain, at any given order in the expansion of the equation of state. At the leading order, only $\alpha_{\mathbf{K}}$ is important, and the twobody Schrodinger equation is reproduced upon minimization of $E-\mu N$ ( $N$ is the number of fermions). This wave function corresponds to a collection of dimers. At the next order, $\beta$ enters and is adjusted to minimize energy. It is consequently found that $\beta$ satisfies the 4-body Schrodinger equation for the low-energy scattering of two molecules at the leading order. We thus use the solution to the latter equation to construct $\beta$ approximately [the result is shown in Eq. 8 below]. In the 
process $\alpha_{\mathbf{K}}$ is computed to a higher order, to minimize energy at this higher level of approximation. Summing up all the contributions to energy up to this level (using the results for $\alpha$ and $\beta$ that one just obtained), one deduces a mean-field interaction between dimers; note that here one derives the correct $a_{d d}$ in accordance with Ref. [7] in such interaction term.

At the third order, $\gamma_{\mathbf{K}_{1}+\mathbf{q} / 4, \cdots, \mathbf{K}_{4}+\mathbf{q} / 4, \mathbf{k}^{\prime}-\mathbf{q} / 2 \uparrow,-\mathbf{k}^{\prime}-\mathbf{q} / 2 \downarrow}$ now appears. This order corresponds to the usual Lee-Yang term [11] for point bosons, although here the derivation is for the molecular boson case. Similarly, $\alpha_{\mathbf{K}}$ and $\beta$ are recomputed to yet a higher order of accuracy. Corrections to the latter are now predominantly at small $q\left[\sim O\left(\sqrt{n_{d} a_{d d}}\right)\right]$.

At this third order, the function $\beta$ in Eq. (3) unifies the Bogoliubov theory of composite bosons and the exact 4-fermion calculations of Ref. [7]. We show this as follows by deducing an asymptotic formula from Bogoliubov theory for composite bosons. We introduce $b_{\mathbf{q}}^{\dagger}=\sum_{\mathbf{k}} \phi_{\mathbf{k}} c_{\mathbf{q} / 2+\mathbf{k} \uparrow}^{\dagger} c_{\mathbf{q} / 2-\mathbf{k} \downarrow}^{\dagger}$, where $\phi_{\mathbf{k}}$ is the internal wave function of the dimer, satisfying $\sum_{\mathbf{k}}\left|\phi_{\mathbf{k}}\right|^{2}=1$. With this substitution, it follows that

$$
\beta_{\mathbf{q} / 2+\mathbf{k} \uparrow, \mathbf{q} / 2-\mathbf{k} \downarrow,-\mathbf{q} / 2+\mathbf{k}^{\prime} \uparrow,-\mathbf{q} / 2-\mathbf{k}^{\prime} \downarrow} \approx x_{\mathbf{q}} \phi_{\mathbf{k}} \phi_{\mathbf{k}^{\prime}}
$$

In fact, by strictly applying the energy minimization principle alone, without resorting to the picture of bosons, we are able to derive the same formula as above (under the condition $q \ll 1 / r_{d}$ and $q \ll\left|\mathbf{k}-\mathbf{k}^{\prime}\right|$, where $r_{d} \sim a$ is the molecular size), and our formula for $x_{\mathbf{q}}$ coincides, at the leading order, with that of a traditional Bose gas [11] whose scattering length equals $a_{d d}$. For $1 / r_{d} \gg q \gg \sqrt{n_{d} a_{d d}}$ and $\left|\mathbf{k}-\mathbf{k}^{\prime}\right| \gg q$, our theory yields the standard asymptotic formula for $x_{\mathbf{q}}$ to obtain

$$
\beta_{\mathbf{q} / 2+\mathbf{k} \uparrow, \mathbf{q} / 2-\mathbf{k} \downarrow,-\mathbf{q} / 2+\mathbf{k}^{\prime} \uparrow,-\mathbf{q} / 2-\mathbf{k}^{\prime} \downarrow}=-\frac{4 \pi n_{d} a_{d d}}{q^{2}} \phi_{\mathbf{k}} \phi_{\mathbf{k}^{\prime}}
$$

plus higher order corrections.

Now let us compute the related behavior which derives from the nonperturbative four-body physics of Ref. [7]. When $q$ and $\left|\mathbf{k}-\mathbf{k}^{\prime}\right|$ are both $\gg \sqrt{n_{d} a_{d d}}$, it can be shown that

$$
\begin{aligned}
& \beta_{\mathbf{q} / 2+\mathbf{k} \uparrow, \mathbf{q} / 2-\mathbf{k} \downarrow},-\mathbf{q} / 2+\mathbf{k}^{\prime} \uparrow,-\mathbf{q} / 2-\mathbf{k}^{\prime} \downarrow \\
&= N_{d} \phi_{\mathbf{q} / 2+\mathbf{k} \uparrow, \mathbf{q} / 2-\mathbf{k} \downarrow,-\mathbf{q} / 2+\mathbf{k}^{\prime} \uparrow,-\mathbf{q} / 2-\mathbf{k}^{\prime} \downarrow}^{(4)}
\end{aligned}
$$

plus higher order corrections. Here $N_{d}=N / 2$, and $\phi^{(4)}$ is the momentum representation of the four-fermion wave function, describing the low-energy scattering of two dimers.

In coordinate space, such a four-fermion wave function takes the asymptotic form $\propto\left(1-a_{d d} / R_{d d}\right) \phi(\mathbf{r}) \phi\left(\mathbf{r}^{\prime}\right)$, when the distance between dimers $R_{d d} \gg r_{d}$. Translating this longdistance boundary condition into momentum space, we get:

$$
\begin{aligned}
& \phi_{\mathbf{q} / 2+\mathbf{k} \uparrow, \mathbf{q} / 2-\mathbf{k} \downarrow,-\mathbf{q} / 2+\mathbf{k}^{\prime} \uparrow,-\mathbf{q} / 2-\mathbf{k}^{\prime} \downarrow}^{(4)} \\
& \approx\left(\delta_{\mathbf{q}, 0}-\frac{4 \pi a_{d d}}{\Omega q^{2}}\right) \phi_{\mathbf{k}} \phi_{\mathbf{k}^{\prime}}, \quad q \ll 1 / r_{d} .
\end{aligned}
$$

where $\left|\mathbf{k}-\mathbf{k}^{\prime}\right| \gg q$, and $\Omega$ is the volume.
Note that Eq. 6 is valid in the phase space region in which $q \ll 1 / r_{d},\left|\mathbf{k}-\mathbf{k}^{\prime}\right|$, while Eq. [8] is valid in the region $q,\left|\mathbf{k}-\mathbf{k}^{\prime}\right| \gg \sqrt{n_{d} a_{d d}}$. Now consider the region of overlap, in which $1 / r_{d} \gg q \gg \sqrt{n_{d} a_{d d}}$ and $\left|\mathbf{k}-\mathbf{k}^{\prime}\right| \gg q$. In this region, both the composite-boson formula [Eq. [6] and the 4fermion formula [Eq. (8)] yield exactly the same result, Eq. (7) above. Thus the conventional picture of many bosons (whose condensate suffers from a quantum depletion, as shown by the function $x_{\mathbf{q}}$ ) and the nonperturbative four-fermion effects are merged into a single picture.

Finally, we turn to experimental implications of this approach closer to unitarity. Here we introduce higher order terms in the equation of state [Eq. (5)] in a phenomenological manner. Our ansatz, for which we have some microscopic support, builds on earlier exact results [19, 20] for non-composite, or true bosons. Thus we presume that

$$
\begin{aligned}
E / \Omega= & n_{d} E_{d}^{(0)}+2 \pi \hbar^{2} n_{d}^{2} a_{d d} / m_{d} \\
& \times(1+4.8144 \sqrt{g}+19.6539 g \ln g+C g)
\end{aligned}
$$

plus higher order terms which we shall omit below. Here $g \equiv$ $n_{d} a_{d d}^{3}$ is the Bose gas parameter. The coefficient in front of $g \ln g$ was first found by Wu for the hard sphere Bose gas [19], and was later shown to be valid for all gases of identical noncomposite bosons.

The phenomenological term containing the unknown parameter $C$ is roughly of the same order of magnitude as the Wu term. Physically, the $C$-term must involve a three-dimer scattering process. This corresponds to a quantum six-body problem, making $C$ very difficult to determine directly from theory. For a dilute gas of bosonic atoms with large positive scattering length, a quantum three-body calculation [21] yields $C \approx 141$. However, this latter result is not applicable to the BEC-BCS crossover system we study here.

To proceed, we calculate $C$ from currently available experimental data, on the radial breathing mode frequencies [12] and axial cloud sizes [8] of cigar-shaped two-component Fermi gases of ${ }^{6} \mathrm{Li}$ atoms in harmonic traps. The atomic scattering length $a$ is taken from Ref. [22] where it is found to diverge at $B=834 \mathrm{G}$. We presume $a_{d d}=0.6 a$ [7]. We start from Eq. [10, and evaluate the axial size (using the local density approximation for the density profile) and the radial breathing mode frequency (using the scaling ansatz of Ref. [23]). The parameter $C$ is then adjusted to best reflect the general features of the data. In this way we arrive at a phenomenological equation of state which extrapolates from deep in the BEC (where it is well controlled) into the range of $k_{F} a$ accessible by experiment.

In Fig. 11 we plot the normalized cloud size $\zeta$ as a function of $B$. In Fig. 2] we plot the radial breathing mode frequency as a function of $1 / k_{F} a$. In both figures, $k_{F}$ is the Fermi wave vector of the noninteracting Fermi gas at the trap center. A value $C=40$ reproduces the trends of the first generation radial mode frequencies [12] and is also not inconsistent with the cloud size data [8]. From the cloud size data, we observe that there may be some uncertainty in $C$ of the order \pm 10 , 


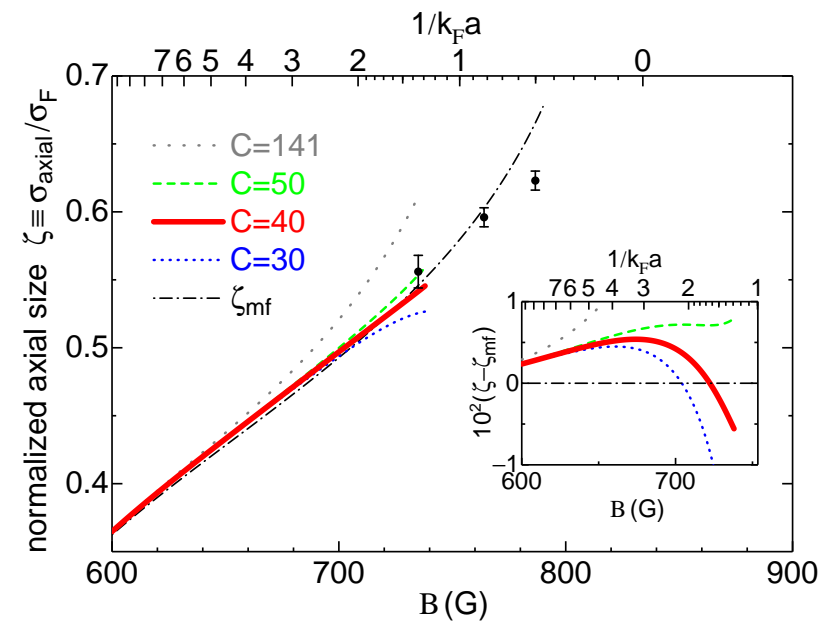

FIG. 1: (color online). Axial cloud size $\sigma_{\text {axial }}$ normalized by that of the noninteracting Fermi gas (with the same number of atoms), $\sigma_{F}$, for a cigar-shaped trapped Fermi gas of ${ }^{6} \mathrm{Li}$ atoms. Error bars: data in [8]. Solid line: Eq. [10] with $C=40$. Dashed line: $C=50$. Dotted line: $C=30$. Sparse dotted line: $C=141$, as in an atomic BEC with large scattering length [21]. Dot-dashed line: bosonic meanfield approximation, as in [8]. In the inset are plotted the differences between the various cloud size curves and the bosonic mean-field curve.



FIG. 2: (color online). Radial breathing mode frequency (normalized by the radial trap frequency) versus $1 / k_{F} a$. Error bars: data from [12]. Solid line: Eq. [10] with $C=40$. Dashed line: $C=50$. Dotted line: $C=30$. Sparse dotted line: $C=141$, as in an atomic BEC with large scattering length [21]. Dot-dashed line: bosonic mean-field approximation. Dot-dot-dashed line: Monte-Carlo simulations [24]. The solid line is extended to indicate the likely continuous curve consistent with data, however there is no analytic formula for this extension. " $\Delta$ " shows the $1 / k_{F} a$ value at which the frequency shift changes sign, for $C=40$. In the inset are plotted the differences between various curves and the bosonic mean-field value. so in both Fig. 1 and Fig. 2 we plot $C=30$ and $C=50$ for comparison. This value of $C$ should be compared with an atomic $\mathrm{BEC}$ with large scattering length, for which $C \approx 141$ [21].

Monte-Carlo (MC) simulations [24, 25] yield a curve for the radial breathing-mode frequencies significantly higher than the published experimental data [12]. However, there are currently "word of mouth" indications that a new generation of breathing mode experiments from the Innsbruck group is in good agreement with MC data. If we use the MC results to deduce $C$, we obtain $90<C<120$ (where our result is most highly constrained by the MC point [25] at $1 / k_{F} a=1$ ). By contrast, the range of acceptable values from the cloud size experiment is $40<C<65$ (where our result is most highly constrained by the data point at $B=735 \mathrm{G}$ in Fig. 1). That there is no overlap might be of some concern. However, it can be shown that the MC data itself [25] is not entirely consistent (within error bars) with the cloud size data [8]. This is not unexpected since these Monte Carlo simulations, which are based on the fixed-node approximation [25] establish an upper bound to the equation of state; at the same time, it is quite plausible that these first generation cloud size experiments may be revised in due time.

It should be stressed, finally, that the range of validity of our low-density formula is, strictly speaking, outside the range of fields accessed in the data which are closer to unitarity. Future experiments which penetrate more deeply into the low density regime will be needed to determine the parameter $\mathrm{C}$ in more detail. As a parenthetical observation, one can see by comparing both figures that the curves associated with different values of $\mathrm{C}$ in the cloud size experiments show a smaller deviation from each other, than do the curves associated with the collective modes. This is because the cloud size is proportional to the 1/5-th power of the dimer-dimer scattering length [8] and is thus less sensitive to the details of the equation of state.

In conclusion the central contribution of our paper is the proposal for a new many-body ground state wave function of the atomic Fermi gas with BEC-BCS crossover. This wave function yields a ground state energy consistent with the correct dimer-dimer scattering length $a_{d d}$ in the BEC regime [7]. It also reproduces the Lee-Yang term, whose numerical value is also fixed by $a_{d d}$. We have also examined the next term beyond the Lee-Yang correction in a phenomenological fashion, building on cloud size data and collective mode experiments. One has to view this phenomenological analysis with some caution since experiments are performed close to unitarity whereas the theory presented here is most appropriate in the deep BEC. More experiments on the cloud size and collective mode frequencies in the deep BEC regime will be required to narrow down a range of the parameter $\mathrm{C}$ which appears in our theory, and thus determine the appropriate equation of state. In the present paper we have had to make due with experiments close to unitarity and with word of mouth information which indicates that the measured radial breathing mode frequencies are in a state of flux, and tending to now 
yield good agreement with the Monte Carlo data. With that proviso the value of $C$ can be as small as 40 and as large as 120. Finally, we stress that there is a distinction here between a BEC of fermionic dimers and an atomic Bose gas with large scattering length where it has been shown [21] that in the latter $C \approx 141$.

Note Added- After this work was completed we became aware of a later related study by Levinsen and Gurarie [26] which produced the correct mean field term in the equation of state, but which did not address the Lee-Yang term, and the other corrections considered here.

This work is partially supported by NSF under NSFMRSEC Grant No. DMR-0213745. We thank J. E. Thomas and C. Chin, as well as Q. Chen, E. Braaten, A. Bulgac, G. Shlyapnikov and D. S. Petrov for helpful communications. We thank anonymous referees of this manuscript for some suggestions.

[1] K. M. O'Hara, S. L. Hemmer, M. E. Gehm, S. R. Granade, and J. E. Thomas, Science 298, 2179 (2002); M. Greiner, C. A. Regal, and D. S. Jin, Nature 426, 537 (2003); S. Jochim, M. Bartenstein, A. Altmeyer, G. Hendl, S. Riedl, C. Chin, J. Hecker Denschlag, and R. Grimm, Science 302, 2101 (2003); M. W. Zwierlein, C. A. Stan, C. H. Schunck, S. M. F. Raupach, S. Gupta, Z. Hadzibabic, and W. Ketterle, Phys. Rev. Lett. 91, 250401 (2003); T. Bourdel, L. Khaykovich, J. Cubizolles, J. Zhang, F. Chevy, M. Teichmann, L. Tarruell, S. J. J. M. F. Kokkelmans, and C. Salomon, Phys. Rev. Lett. 93, 050401 (2004); C. Chin, M. Bartenstein, A. Altmeyer, S. Riedl, S. Jochim, J. Hecker Denschlag, and R. Grimm, Science 305, 1128 (2004); J. Kinast, A. Turlapov, J. E. Thomas, Q. Chen, J. Stajic, and K. Levin, Science 307, 1296 (2005).

[2] J. Kinast, S. L. Hemmer, M. E. Gehm, A. Turlapov, and J. E. Thomas, Phys. Rev. Lett. 92, 150402 (2004); M. Bartenstein, A. Altmeyer, S. Riedl, S. Jochim, C. Chin, J. Hecker Denschlag, and R. Grimm, Phys. Rev. Lett. 92, 203201 (2004).

[3] M. W. Zwierlein, J. Abo-Shaeer, A. Schirotzek, C. Schunck, and W. Ketterle, Nature 435, 1047 (2005).

[4] D. M. Eagles, Phys. Rev. 186, 456 (1969).
[5] A. J. Leggett, Modern Trends in the Theory of Condensed Matter (Springer-Verlag, Berlin, 1980), pp.13-27.

[6] Qijin Chen, J. Stajic, Shina Tan, and K. Levin, Phys. Rep. 412, 1 (2005); Qijin Chen, J. Stajic, and K. Levin, cond-mat/0508603.

[7] D. S. Petrov, C. Salomon and G. V. Shlyapnikov, Phys. Rev. Lett. 93, 090404(2004); see also related work in Refs. [26, 27] which reproduces the correct four-fermion physics and the value of $a_{d d}$.

[8] M. Bartenstein, A. Altmeyer, S. Riedl, S. Jochim, C. Chin, J. H. Denschlag, and R. Grimm, Phys. Rev. Lett. 92, 120401 (2004).

[9] M. J. Holland, C. Menotti, and L. Viverit, cond-mat/0404234 (2004).

[10] P. Pieri and G. C. Strinati, Phys. Rev. B 61, 15370 (2000).

[11] T. D. Lee and C. N. Yang, Phys. Rev. 105, 1119 (1957); T. D. Lee, K. Huang, and C. N. Yang, Phys. Rev. 106, 1135 (1957).

[12] J. Kinast, A. Turlapov, and J. E. Thomas, Phys. Rev. A 70, 051401(R) (2004).

[13] Shina Tan, cond-mat/0510055.

[14] Here "spin" refers to one of two internal states of the fermion.

[15] P. Pieri, L. Pisani, and G. C. Strinati, Phys. Rev. B 72, 012506 (2005).

[16] H. Hu, X.-J. Liu, and P. D. Drummond, Euro. Phys. Lett. 74, 57 (2006).

[17] S. Stringari, Europhys. Lett. 65, 749 (2004).

[18] For s-wave contact interactions, $a_{d d}=0.6 a$ [7] is not an independent length scale.

[19] T. T. Wu, Phy. Rev. 115, 1390 (1959).

[20] N. M. Hugenholtz and D. Pines, Phys. Rev. 116, 489 (1959).

[21] E. Braaten, H.-W. Hammer, and T. Mehen, Phys. Rev. Lett. 88, 040401 (2002).

[22] M. Bartenstein, A. Altmeyer, S. Riedl, R. Geursen, S. Jochim, C. Chin, J. H. Denschlag, R. Grimm, A. Simoni, E. Tiesinga, et al., Phys. Rev. Lett. 94, 103201 (2005).

[23] H. Hu, A. Minguzzi, X.-J. Liu, and M. P. Tosi, Phys. Rev. Lett. 93, 190403 (2004).

[24] G. E. Astrakharchik, R. Combescot, X. Leyronas, and S. Stringari, cond-mat/0503618 (2005).

[25] G. E. Astrakharchik, J. Boronat, J. Casulleras, and S. Giorgini, Phys. Rev. Lett. 93, 200404 (2004).

[26] J. Levinsen and V. Gurarie, Phys. Rev. A 73, 053607 (2006).

[27] I. V. Brodsky, M. Y. Kagan, A. V. Klaptsov, R. Combescot, and X. Leyronas, Phys. Rev. A 73, 032724 (2006). 